\title{
Bovine colostrum supplementation attenuates the decrease of salivary lysozyme and enhances the recovery of neutrophil function after prolonged exercise
}

\author{
Glen Davison* and Bethany C. Diment \\ Department of Sport and Exercise Science, Aberystwyth University, Carwyn James Building, Penglais Campus, Aberystwyth, \\ Ceredigion SY23 3FD, UK \\ (Received 28 July 2009 - Revised 17 November 2009 - Accepted 18 November 2009 - First published online 24 December 2009)
}

Oral supplementation with bovine colostrum (COL) has been shown to enhance immunity in human subjects. However, there is limited research on the use of bovine COL supplementation to counter exercise-induced immunodepression, as a model of stress-induced immunodepression, and previous research has focused primarily on salivary IgA. The aim of the present study was to determine the effects of bovine COL supplementation on exercise-induced changes in innate immunity (neutrophil function and salivary lysozyme) in addition to salivary IgA. Twenty healthy, active men cycled for $2 \mathrm{~h}$ at approximately $64 \%$ maximal oxygen uptake after 4 weeks of daily bovine COL ( $n$ 10) or placebo (PLA, $n$ 10) supplementation. Blood and saliva samples were obtained before and after supplementation, before and after exercise. Exercise induced significant increases in markers of physiological stress and stress to the immune system (circulating neutrophils, neutrophil:lymphocyte ratio, immature granulocytes, atypical lymphocytes and plasma cortisol), but there were no differences between the COL and PLA groups. Significant group $\times$ time interactions (two-way mixed model ANOVA) were observed for neutrophil function (stimulated degranulation) and salivary lysozyme concentration and release $(P<0 \cdot 05)$. Significant exercise-induced decreases were observed in these parameters, and bovine COL supplementation either speeded the recovery (neutrophil function) or prevented the decrease (salivary lysozyme) in these measures of innate immunity. These results suggest that 4 weeks of bovine COL supplementation limits the immunodepressive effects induced by an acute prolonged physical stressor, such as exercise, which may confer some benefits to host defence.

Mucosal immunity: Innate immunity: Host defence: Saliva

Prolonged exercise, which is often used as a model of physical stress, causes acute depression of many components of the immune system ${ }^{(1-6)}$. Exercise is an excellent, controllable, quantifiable and reproducible model with which to study the effects of stress, and physical stress in particular, on immunity ${ }^{(2,3,6)}$. Numerous studies have investigated the effectiveness of various nutritional strategies and supplementation at minimising immune perturbations following prolonged exercise, but limited studies have investigated the effects of supplementation with bovine colostrum (COL). Bovine COL, the initial milk produced by cows in the first $48 \mathrm{~h}$ after parturition, contains bioactive components that play significant roles within the immune system (such as immunoglobulins and antimicrobial proteins) in addition to the macronutrients and micronutrients found in milk $^{(7,8)}$. The importance of bovine COL to assist in the protection against infection for newborn calves is well known and has led to interest in the potential immunological benefits of bovine $\mathrm{COL}$ as a nutritional supplement in human subjects ${ }^{(7)}$. It has been demonstrated that bovine COL can enhance the humoral immune response to an orally administered vaccine ${ }^{(9)}$, which is also supported by its modulatory effects on cell-mediated immunity ${ }^{(10)}$. These results suggest that bovine COL supplementation may also be of benefit to individuals who are subjected to acutely depressed immune function (i.e. following a physical stressor such as endurance exercise). Despite this, few studies have examined the effects of bovine COL supplementation on immune function in a physical stress model. Recently, it has been demonstrated that a 12-week supplementation intervention in a group of distance runners resulted in a $79 \%$ increase in resting salivary $\operatorname{IgA}^{(11)}$. A $33 \%$ increase in salivary IgA has also been found after only 2 weeks of supplementation ${ }^{(12)}$. It has been proposed that an increase in resting salivary $\operatorname{IgA}$ may attenuate exercise-induced perturbations in salivary IgA and aid in the protection against developing upper respiratory tract infection ${ }^{(13)}$. However, it is also important to study other components of the immune system and host defence. In a recent study, Shing et al. ${ }^{(14)}$ investigated the effects of bovine COL supplementation on salivary $\operatorname{IgA}$ in addition to some components of innate immunity. They observed some minor effects suggestive of enhanced neutrophil function (CD89 surface receptor expression) at rest but not following

Abbreviations: COL, colostrum; PLA, placebo; Post-Ex, samples taken immediately after $2 \mathrm{~h}$ exercise bout; $1 \mathrm{~h}$ Post-Ex, samples taken after $1 \mathrm{~h}$ of post-exercise recovery; Pre-Ex, samples taken before $2 \mathrm{~h}$ exercise bout.

* Corresponding author: Glen Davison, fax +44 1970 628557, email gdd@aber.ac.uk 
exercise in the COL-supplemented group. However, leucocyte surface receptor expression has limited value as a marker of human immune modulation ${ }^{(15)}$, and a measure of actual neutrophil function would be valuable. Furthermore, Shing et al. ${ }^{(14)}$ observed limited changes in the neutrophil receptor expression markers, even in the placebo (PLA) condition, giving little scope for COL supplementation to be of benefit.

The underlying mechanism responsible for the effect of bovine COL on human immune parameters remains unclear; however, evidence from animal studies suggests that it may stimulate leucocyte capacity, specifically by modulating neutrophil phagocytosis and 'killing' capacity ${ }^{(16,17)}$. Therefore, the aims of the present study were to determine the effects of bovine COL supplementation on blood neutrophil functional capacity and salivary lysozyme release (in addition to $\operatorname{IgA}$ ) after a bout of endurance exercise that is known to cause significant acute depression of innate immunity.

\section{Experimental methods}

The present study was conducted according to the guidelines laid down in the Declaration of Helsinki and all procedures were approved by the Aberystwyth University Research Ethics Committee. Written informed consent was obtained from all subjects. Subjects also completed a pre-exercise screening questionnaire (physical activity readiness questionnaire) before participating in each test.

\section{Subjects}

Twenty healthy recreationally active men (age 25 (SD 5) years, body mass 78.5 (SD 9.0) kg, $\mathrm{V}_{\mathrm{O} 2 \max }, 52.2$ (SD 7.7) $\mathrm{ml} / \mathrm{min}$ per $\mathrm{kg}$ ) participated in the present study. Subjects were randomly assigned to one of two groups, either PLA supplementation ( $n$ 10) or bovine COL supplementation (n 10). Supplementation was double blind, and subjects in the COL group received oral supplementation with bovine COL $(20 \mathrm{~g} / \mathrm{d})$, whereas the PLA group received a PLA containing an isoenergetic (approximately $300 \mathrm{~kJ}$ per $20 \mathrm{~g}$ powder) and isomacronutrient mixture of milk protein concentrate $(23 \%)$ and skimmed milk powder $(77 \%)$. Approximate macronutrient composition was $52 \%$ protein, $37 \%$ carbohydrate (lactose) and $0.8 \%$ fat for the bovine COL product, and $50 \%$ protein, $41 \%$ carbohydrate (lactose) and $0.8 \%$ fat for the PLA. Supplements were taken daily for $28 \mathrm{~d}$ in accordance with the manufacturer's instructions for the COL product. COL was provided by Neovite UK, London.

\section{Testing protocols}

All subjects completed three exercise bouts; a preliminary trial ( $\mathrm{V}_{\mathrm{O} 2 \max }$ determination), a familiarisation trial and a main trial. For $\mathrm{V}_{\mathrm{O} 2 \max }$ determination, subjects performed a continuous incremental test $(30 \mathrm{~W} / \mathrm{min}$ ramp rate) to volitional exhaustion on an electrically braked cycle ergometer (Lode Excalibur, Groningen, The Netherlands) as previously described ${ }^{(18)}$. Heart rate and rating of perceived exertion were recorded during this period using a telemetric device (Polar, Kempele, Finland) and the Borg Scale, respectively. This test took place at approximately day 14 of the study period (where day 0 is the day on which baseline samples were obtained: see below). The familiarisation trial was designed to familiarise subjects to the testing procedures and ensure that the correct exercise intensity had been identified from the $\mathrm{V}_{\mathrm{O} 2 \mathrm{max}}$ test. This test took place on approximately day 21 and was completed at least $6 \mathrm{~d}$ before the main trial. The familiarisation trial was 60 min duration.

\section{Main trials}

Subjects were instructed to consume $500 \mathrm{ml}$ of water $2 \mathrm{~h}$ pre-exercise before arrival at the laboratory on the morning of the main trial. All subjects were requested to arrive between 09.00 and 09.30 hours, after an overnight fast of at least $10 \mathrm{~h}$. Subjects sat quietly in the laboratory for 20-30 min before beginning exercise for $2 \mathrm{~h}$ at an intensity of approximately $64 \% \mathrm{~V}_{\mathrm{O} 2 \max }$. To ensure the relative exercise intensity was equivalent between subjects, the precise exercise intensity was determined relative to each subject's individual gas exchange threshold and their $\mathrm{V}_{\mathrm{O} 2 \max }$ exercise intensity. The relative intensity was set at $20 \%$ of the difference between gas exchange threshold and $\mathrm{V}_{\mathrm{O} 2 \max }(20 \% \Delta)$. Expired gas was analysed during the 15th, 30th, 60th, 90th and 120th min of exercise. Heart rate and rating of perceived exertion were recorded every $15 \mathrm{~min}$ during exercise. Subjects were given $5 \mathrm{ml} / \mathrm{kg}$ body mass of a beverage (a low energy lemon flavoured squash with artificial sweetener, providing $21 \mathrm{~kJ}$ energy, $0 \cdot 1 \mathrm{~g}$ protein, $1 \cdot 1 \mathrm{~g}$ carbohydrate, trace fat and trace sodium per litre of solution), before exercise, followed by $2.5 \mathrm{ml} / \mathrm{kg}$ body mass every $15 \mathrm{~min}$ during exercise. This was equivalent to approximately 1.8 litre of beverage, on average, over the course of the $2 \mathrm{~h}$ trial.

Subjects completed a weighed food record diary for the $24 \mathrm{~h}$ period before the main trial. On the day of the main trial, they also completed a FFQ relating to the previous $7 \mathrm{~d}$. The dietary records were analysed using computer dietary analysis software (CompEat, Nutrition Systems, London, UK). Subjects were all non-smokers and were required to abstain from alcohol, caffeine and strenuous activity for $48 \mathrm{~h}$ before the main trial. It was also stipulated that subjects should not take any other supplement (including vitamin, mineral or 'sports' supplements) during and for the 4 weeks before the study.

\section{Blood samples}

Blood samples were obtained by venepuncture, with minimal stasis, from an antecubital vein and collected into vacutainer tubes $\left(4.5 \mathrm{ml}\right.$ into a $\mathrm{K}_{3}$ EDTA-treated tube and $7 \mathrm{ml}$ into a heparin-treated tube). An initial baseline (Baseline) sample was taken in the morning, after an overnight fast, before beginning the supplementation period. Further venous blood samples were taken immediately before beginning the $2 \mathrm{~h}$ exercise bout (Pre-Ex), within a few minutes of completing the exercise bout (Post-Ex) and after $1 \mathrm{~h}$ of recovery $(1 \mathrm{~h}$ Post-Ex), during which subjects remained in the laboratory and were allowed to undertake restful activities such as reading or using a computer. All blood samples were obtained while subjects were in the seated position. Subjects were asked to sit without changes of posture and minimal movement for $10 \mathrm{~min}$ before all blood samples were drawn, except the Post-Ex sample, which was drawn as soon as possible after completing the exercise (within $3 \mathrm{~min}$ ). 


\section{Saliva samples}

Saliva samples were collected as previously described ${ }^{(18)}$, at Baseline, Pre-Ex, Post-Ex and $1 \mathrm{~h}$ Post-Ex. Briefly, timed, unstimulated whole saliva was obtained while subjects were seated, with the head tilted slightly forward and making minimal orofacial movement. They were asked to swallow to empty the mouth, before whole saliva was collected by passive dribble into a pre-weighed sterile tube for an initial period of $2 \mathrm{~min}$. If insufficient saliva was obtained after the initial $2 \mathrm{~min}$ period, the process was repeated. Subjects were not allowed to drink for at least $10 \mathrm{~min}$ before each sample. The tube was reweighed after collection of the sample so that saliva volume (and hence flow rate) could be estimated. Tubes were weighed to the nearest $0.1 \mathrm{mg}$ (saliva density was assumed to be $1.00 \mathrm{~g} / \mathrm{ml}$ ) and aliquots were frozen, at $-80^{\circ} \mathrm{C}$, for later analysis.

\section{Analytical methods}

Blood analysis. Haematological analysis was performed on the blood samples collected into the $\mathrm{K}_{3}$ EDTA tubes using an automated haematology analyser (ABX Pentra 120, Horiba Medical, Montpellier, France). Blood $\mathrm{Hb}$ concentration, haematocrit, total and differential leucocyte counts were measured. Haematocrit was also determined by standard microcentrifugation (with a Hawksley microcentrifuge) for use, along with $\mathrm{Hb}$ concentration, to estimate post-exercise changes in blood and plasma volume (compared to the Pre-Ex sample) using the equations of Dill \& Costill ${ }^{(19)}$. Blood lactate and glucose concentrations were determined in heparinised blood using an automated analyser (YSI 2300 Stat Plus, Yellow Springs, OH, USA). Blood haematocrit analyses by microcentrifugation were carried out in triplicate.

A $1 \mathrm{ml}$ aliquot of blood from the heparin tube was used for measurement of neutrophil degranulation (see below). The remaining blood was centrifuged at $1500 \mathrm{~g}$ for $10 \mathrm{~min}$ at $4^{\circ} \mathrm{C}$, and aliquots of plasma were stored at $-80^{\circ} \mathrm{C}$ for later analysis of cortisol ( $\mathrm{K}_{3}$ EDTA tube) and elastase (heparin tube) concentrations. Plasma cortisol concentration was determined in duplicate using a commercially available ELISA kit (DRG, Lahn, Germany). All samples were thawed only once before analysis.

In vitro stimulated neutrophil degranulation. The neutrophil degranulation response to bacterial stimulant (840-15, Sigma, Poole, UK) was determined as previously described $^{(20)}$. Neutrophil degranulation was expressed as the amount of stimulated elastase release per neutrophil. Elastase concentration was determined in duplicate using a commercially available ELISA kit (Merck Calibiochem, Darmstadt, Germany).

Salivary analysis. All samples were thawed only once before analysis. After thawing, at room temperature, samples were centrifuged at $15000 \mathrm{~g}$ for $2 \mathrm{~min}$ to pellet debris, precipitate mucins and obtain a clear supernatant. Saliva osmolality was determined using a freezing point depression osmometer (Osmomat 030, Gonotec, GbBH, Berlin, Germany) calibrated with $300 \mathrm{mOsmol} / \mathrm{kg}$ saline solution, in accordance with the manufacturer's instructions. Accuracy was confirmed with a $50 \mathrm{mOsmol} / \mathrm{kg}$ reference solution. Saliva samples were screened for blood contamination by the measurement of salivary transferrin concentration. Blood contamination was assumed if salivary transferrin concentration exceeded $10 \mathrm{mg} / \mathrm{l}$. In the event that blood contamination was detected, all salivary data for that subject were excluded from the analysis.

Salivary IgA, lysozyme and transferrin. Aliquots of saliva were analysed for the determination of $\operatorname{IgA}$, transferrin (Salimetrics, State College, PA, USA) and lysozyme (Biomedical Technologies Inc., Stoughton, MA, USA) in duplicate using commercially available ELISA kits.

\section{Data analysis}

Statistical analyses were carried out using the statistical computer software package SPSS (v17.00; SPSS Inc., Chicago, IL, USA). Blood glucose concentration, immature granulocyte count, stimulated neutrophil degranulation, plasma cortisol concentration, salivary $\operatorname{IgA}$ concentration, IgA:osmolality ratio, IgA secretion rate and lysozyme:osmolality ratio data were normally distributed. The other variables were not normally distributed but were normalised with log transformation (neutrophil count, lymphocyte count, neutrophil:lymphocyte ratio, atypical lymphocyte count and salivary lysozyme concentration) or square root transformation (salivary lysozyme secretion rate and blood lactate concentration) before analysis. To compare the two independent groups' responses to exercise a two-way mixed model (betweengroups repeated measures), ANOVA (group $\times$ time) was used. Post hoc independent $t$ tests with the Holm-Bonferroni correction were used, where appropriate, to compare time point-specific differences between groups. When there was evidence of an interaction, the effect of time was analysed in each group independently, with one-way repeatedmeasures ANOVA and post hoc paired $t$ tests with the Holm-Bonferroni correction, where appropriate. The Greenhouse-Geisser correction was applied to correct for violations of the assumption of sphericity, where necessary. All results are presented as means and standard deviations.

\section{Results}

There was no difference between groups' demographic data, dietary composition during the $48 \mathrm{~h}$ before the main trial (data not shown) or relative exercise intensity during the main trial. There was no differences between groups in mean heart rate (overall mean, 145 (SD 12) beats per minute), subjective rating of perceived exertion (overall, 14 (SD 2)) or mean percentage of $\mathrm{V}_{\mathrm{O} 2 \max }$ (overall, 64.3 (SD 5.7)\%) during the main trial (independent $t$ test, $P=0.699,0.752$ and $0 \cdot 882$, respectively).

The dietary analysis (both the weighed food record and FFQ) also confirmed no deficiencies or excessive intake of any nutrient in all subjects. Estimates of blood volume change were similar between groups Post-Ex (4.5 (SD 1.9)\% and 5.8 (SD 2.6) \% decrease for PLA and COL, respectively) and $1 \mathrm{~h}$ Post-Ex (1.8 (SD 5.5)\% and 1.1 (SD 3.2)\% decrease, respectively) with no difference between groups (two-way ANOVA group $\times$ time interaction, $P=0.414)$. A similar pattern was evident for estimates of plasma volume change between groups: Post-Ex (3.6 (SD 1.6) \% and 2.4 (SD 2.2)\% decrease, respectively) and $1 \mathrm{~h}$ Post-Ex (1.8 (SD 2.9)\% and 0.2 (SD 2.6) \% decrease, respectively) with no difference 
between groups (two-way ANOVA group $\times$ time interaction, $P=0 \cdot 138)$. Hence, it was not deemed necessary to correct any variables for changes in blood or plasma volume. Furthermore, adjusting for changes in blood and plasma volume did not influence the outcome of any statistical analyses (data not shown).

\section{Blood lactate and glucose responses to exercise}

Blood lactate and glucose concentrations were only measured at Pre-Ex, Post-Ex and $1 \mathrm{~h}$ Post-Ex. There was no difference between groups in the exercise-induced changes in blood lactate concentration (two-way ANOVA group $\times$ time interaction, $P=0.223$ ). There was a significant time effect $(P<0.001)$ with a mean concentration of 0.75 (SD 0.15 ) $\mathrm{mmol} / \mathrm{l}$ Pre-Ex, 1.32 (SD 0.39 ) $\mathrm{mmol} / \mathrm{l}$ Post-Ex (significantly higher than Pre-Ex, $P<0.001$ ) and 0.88 (SD $0 \cdot 24) \mathrm{mmol} / \mathrm{l}$ at $1 \mathrm{~h}$ Post-Ex (significantly higher than Pre-Ex, $P=0.032$ ). There was no difference between groups in the exercise-induced changes in blood glucose concentration (two-way ANOVA group $\times$ time interaction, $P=0.096)$. There was a significant time effect $(P<0 \cdot 001)$ with a mean concentration of 4.4 (SD 0.4) $\mathrm{mmol} / \mathrm{l}$ Pre-Ex, $4 \cdot 1$ (SD 0.4) $\mathrm{mmol} / \mathrm{l}$ Post-Ex (significantly lower than Pre-Ex, $P=0.006)$ and $3.8($ SD $0 \cdot 3) \mathrm{mmol} / \mathrm{l}$ at $1 \mathrm{~h}$ Post-Ex (significantly lower than Pre-Ex, $P<0 \cdot 001)$.

\section{Plasma cortisol concentration}

There was no difference between groups in the exerciseinduced changes in plasma cortisol concentration (two-way ANOVA group $\times$ time interaction, $P=0 \cdot 297)$. There was a significant time effect $(P=0 \cdot 001)$ with a mean concentration of $401(\mathrm{SD} 150) \mathrm{nmol} / \mathrm{l}$ at baseline, 398 (SD 189) nmol/l Pre-Ex, 500 (SD 175) nmol/l Post-Ex (significantly higher than Pre-Ex, $P=0.033$ ) and $343(\mathrm{SD} 128) \mathrm{nmol} / \mathrm{l}$ at $1 \mathrm{~h}$ Post-Ex (not significantly different from Pre-Ex, $P=0 \cdot 314$ ).

\section{Circulating leucocyte number and neutrophil function}

There was no difference between groups in the exerciseinduced changes in circulating neutrophil count, lymphocyte count, neutrophil:lymphocyte ratio, immature granulocytes and atypical lymphocytes (Table 1). Post hoc analysis revealed a significant exercise-induced increase for all of these parameters. Lymphocytes and atypical lymphocytes had returned to pre-exercise counts by $1 \mathrm{~h}$ Post-Ex, whereas neutrophils, neutrophil:lymphocyte ratio and immature granulocytes remained elevated (Table 1).

There was a significant difference between groups in the exercise-induced changes in in vitro stimulated neutrophil degranulation (two-way ANOVA group $\times$ time interaction, $P=0.047)$. One-way ANOVA on each group showed a time effect in the PLA group $(P<0.001)$ and the COL group $(P<0.001)$. Further post hoc analysis showed that there was no difference between Pre-Ex and Baseline in stimulated elastase released per neutrophil $(P=0 \cdot 12)$ in the PLA group. There was a significant decrease below Pre-Ex, at Post-Ex $(P=0.002)$ and $1 \mathrm{~h}$ Post-Ex $(P<0.001)$ in this group (Fig. 1). There was no difference between Pre-Ex and Baseline $(P=0.363)$ in the COL group. There was a significant decrease below Pre-Ex, at Post-Ex $(P=0.003)$ and $1 \mathrm{~h}$ Post-Ex $(P<0.001)$ in this group (Fig. 1). Independent $t$ tests between groups at each time point showed no difference at Baseline $(P>0.999)$. There was a trend for higher stimulated neutrophil degranulation in the COL group at Pre-Ex $(P=0.081)$, but there was no difference at Post-Ex $(P=0.963)$. However, stimulated neutrophil degranulation was significantly higher in the COL group at $1 \mathrm{~h}$ Post-Ex $(P=0 \cdot 018)$.

\section{Salivary analysis}

Blood contamination was detected in the saliva samples from four subjects, two in each group leaving $n 8$ in each group for all saliva results.

Table 1. Differential blood leucocyte counts and salivary parameters

(Mean values and standard deviations for the whole groupt)

\begin{tabular}{|c|c|c|c|c|c|c|c|c|c|}
\hline & \multicolumn{2}{|c|}{ Baseline } & \multicolumn{2}{|c|}{ Pre-Ex } & \multicolumn{2}{|c|}{ Post-Ex } & \multicolumn{2}{|c|}{ 1h Post-Ex } & \multirow{2}{*}{$\begin{array}{l}\text { Two-way ANOVA } \\
\text { main effects, } \\
P=\text { (group; time; } \\
\text { interaction) }\end{array}$} \\
\hline & Mean & SD & Mean & SD & Mean & SD & Mean & SD & \\
\hline \multicolumn{10}{|l|}{ Blood parameters ( $n$ 20) } \\
\hline Neutrophils $\left(\times 10^{9}\right.$ cells/l) & $2 \cdot 9$ & 1.6 & $2 \cdot 7$ & 0.9 & $6 \cdot 8^{*}$ & $2 \cdot 6$ & $7 \cdot 8^{*}$ & 1.9 & $0.868 ;<0.001 ; 0.801$ \\
\hline Lymphocytes $\left(\times 10^{9}\right.$ cells $/$ l) & $2 \cdot 0$ & 0.4 & 1.9 & 0.4 & $2 \cdot 5^{\star}$ & 0.9 & $1 \cdot 8$ & 0.7 & $0.037 ;<0.001 ; 0.557$ \\
\hline Neu:lymph ratio & 1.58 & $1 \cdot 15$ & 1.49 & 0.74 & $2 \cdot 88^{\star}$ & 1.34 & $5 \cdot 06^{\star}$ & $2 \cdot 72$ & $0.160 ;<0.001 ; 0.732$ \\
\hline Immature granulocytes $\left(\times 10^{9}\right.$ cells $/$ l) & 0.02 & 0.01 & 0.01 & 0.01 & $0.06^{\star}$ & 0.03 & $0.06^{*}$ & 0.04 & $0.887 ;<0.001 ; 0.434$ \\
\hline Atypical lymphocytes $\left(\times 10^{9}\right.$ cells/l) & 0.07 & 0.02 & 0.07 & 0.02 & $0 \cdot 10^{*}$ & 0.03 & 0.07 & 0.02 & $0.683 ;<0.001 ; 0.517$ \\
\hline \multicolumn{10}{|l|}{ Salivary parameters $(n 16)$} \\
\hline IgA concentration (mg/l) & 158 & 49 & 128 & 41 & 133 & 47 & 117 & 35 & $0.183 ; 0.009 ; 0.565$ \\
\hline IgA:osmolality ratio $(\mathrm{mg} / \mathrm{mOsmol})$ & $2 \cdot 7^{\star}$ & 0.8 & $2 \cdot 1$ & 0.8 & 1.8 & 0.5 & 1.9 & 0.5 & $0.151 ;<0.001 ; 0.624$ \\
\hline IgA secretion rate $(\mu \mathrm{g} / \mathrm{min})$ & 58.7 & 28.9 & $43 \cdot 3$ & 23.0 & $50 \cdot 4$ & $29 \cdot 1$ & $51 \cdot 1$ & $26 \cdot 7$ & $0.902 ; 0.072 ; 0.756$ \\
\hline Lysozyme:osmolality ratio (mg/mOsmol) & 0.22 & 0.11 & 0.25 & 0.12 & $0 \cdot 13^{*}$ & 0.05 & $0 \cdot 15^{\star \star}$ & 0.07 & $0.977 ; 0.003 ; 0.178$ \\
\hline
\end{tabular}

Pre-Ex, samples taken before $2 \mathrm{~h}$ exercise bout; Post-Ex, samples taken immediately after $2 \mathrm{~h}$ exercise bout; $1 \mathrm{~h}$ Post-Ex, samples taken after $1 \mathrm{~h}$ of post-exercise recovery; Neu:lymph ratio, neutrophil to lymphocyte ratio.

Mean values were significantly different from Pre-Ex: ${ }^{\star} P<0.05,{ }^{\star \star} P<0.01$ (post hoc analysis time effects).

$\dagger$ There were no significant differences between groups (two-way ANOVA interactions, $P>0.05$ ). 


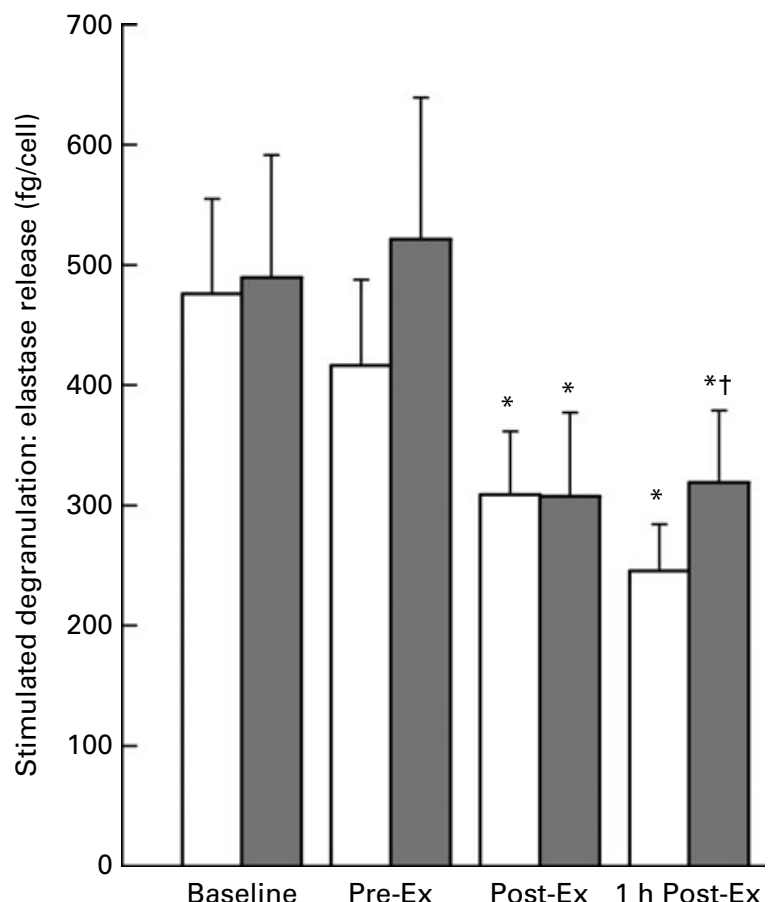

Fig. 1. Neutrophil degranulation. Two-way ANOVA main effects: $P=0.077$ (group); $P<0.001$ (time); $P=0.047$ (group $\times$ time interaction). ${ }^{*}$ Mean values were significantly different from samples taken before $2 \mathrm{~h}$ exercise bout (Pre-Ex) $(P<0.05$; post hoc analysis). † Mean values were different from placebo (PLA, $\square)$ at the same time point between the groups $(P<0.05$; post hoc analysis). Post-Ex, samples taken immediately after $2 \mathrm{~h}$ exercise bout; $1 \mathrm{~h}$ Post-Ex, samples taken after $1 \mathrm{~h}$ of post-exercise recovery; COL, colostrum ( $\square$ ).

Salivary IgA. There was no difference between groups in the exercise-induced changes in salivary IgA concentration (Table 1). There was a significant time effect $(P=0.009)$, but post hoc analysis could not identify any specific differences between time points. There was no difference between groups in the exercise-induced changes in salivary IgA:osmolality ratio (Table 1). There was a significant time effect $(P<0.001)$. Post hoc analysis showed that IgA:osmolality ratio was lower at Pre-Ex than Baseline $(P=0 \cdot 027)$. Salivary IgA:osmolality ratio did not change Post-Ex $(P=0.156)$ and $1 \mathrm{~h}$ Post-Ex $(P=0 \cdot 180)$ compared to Pre-Ex. There was no difference between groups in the exercise-induced changes in salivary $\operatorname{IgA}$ secretion rate (Table 1), and only a trend was evident for a main effect of time $(P=0.072)$.

Salivary lysozyme. Two-way ANOVA showed a significant group $\times$ time interaction for salivary lysozyme concentration $(P=0.044$, Table 2). One-way ANOVA on each group showed a time effect in the PLA group $(P=0.018)$ but not in the COL group $(P=0 \cdot 146)$. Further post hoc analysis showed that there was no difference between PreEx and Baseline $(P=0 \cdot 126)$ in the PLA group. There was a significant decrease below Pre-Ex, at Post-Ex $(P=0.014)$ but not $1 \mathrm{~h}$ Post-Ex $(P=0.075)$ in this group (Table 2). Independent $t$ tests between groups at each time point showed a trend for higher salivary lysozyme at Post-Ex $(P=0.065)$ in the COL group. There was no difference between groups in the exercise-induced changes in salivary lysozyme:osmolality ratio (Table 1), with a significant time effect evident $(P=0.003)$. Post hoc analysis showed no difference in lysozyme:osmolality ratio between Baseline and Pre-Ex $(P=0.141)$ and a decreased below Pre-Ex at Post-Ex $(P=0.004)$ and $1 \mathrm{~h}$ Post-Ex $(P=0.013)$. Two-way ANOVA showed a significant group $\times$ time interaction for salivary lysozyme secretion rate $(P=0.002$, Table 2$)$. Post hoc analysis showed that there was no difference in the resting measures before and after the supplementation period. One-way ANOVA on each group showed a time effect in the PLA group $(P=0.004)$ but not in the COL group $(P=0.373)$. Further post hoc analysis showed that there was no difference between Pre-Ex and Baseline $(P=0 \cdot 122)$ in the PLA group, but a significant decrease below Pre-Ex and at Post-Ex $(P=0.036)$, which recovered by $1 \mathrm{~h}$ Post-Ex $(P=0.301)$.

\section{Discussion}

The main findings of the present study are that 4 weeks of bovine COL supplementation improved the speed of recovery of neutrophil function after exercise-induced immunodepression and reduced exercise-induced alterations in salivary lysozyme concentration and secretion rate. This is the first study to demonstrate that COL supplementation enhances these innate immune parameters in the recovery period after prolonged endurance exercise that causes acute immunodepression. While some previous studies have

Table 2. Salivary lysozyme responses

(Mean values and standard deviations)

\begin{tabular}{|c|c|c|c|c|c|c|c|c|c|}
\hline & \multicolumn{2}{|c|}{ Baseline } & \multicolumn{2}{|c|}{ Pre-Ex } & \multicolumn{2}{|c|}{ Post-Ex } & \multicolumn{2}{|c|}{$1 \mathrm{~h}$ Post-Ex } & \multirow{2}{*}{$\begin{array}{l}\text { Two-way ANOVA main effects, } \\
P=\text { (group; time; interaction) }\end{array}$} \\
\hline & Mean & SD & Mean & SD & Mean & SD & Mean & SD & \\
\hline \multicolumn{10}{|c|}{ Lysozyme concentration (mg/l) } \\
\hline PLA & $10 \cdot 3$ & $7 \cdot 3$ & 13.7 & 7.4 & $5 \cdot 9^{*}$ & $2 \cdot 2$ & 7.5 & 3.8 & \multirow[t]{2}{*}{$0.014 ; 0.244 ; 0.044$} \\
\hline $\mathrm{COL}$ & $11 \cdot 8$ & 3.5 & $12 \cdot 4$ & $5 \cdot 6$ & $13 \cdot 1$ & 7.5 & 9.5 & $6 \cdot 1$ & \\
\hline \multicolumn{10}{|c|}{ Lysozyme secretion rate ( $\mu \mathrm{g} / \mathrm{min})$} \\
\hline PLA & 3.1 & 1.3 & $5 \cdot 1$ & 3.3 & $2 \cdot 4^{*}$ & 1.5 & 4.0 & $2 \cdot 6$ & \multirow[t]{2}{*}{$0.460 ; 0.479 ; 0.002$} \\
\hline $\mathrm{COL}$ & $4 \cdot 2$ & 1.4 & 3.7 & 1.7 & $5 \cdot 1$ & 3.4 & 4.2 & 2.4 & \\
\hline
\end{tabular}

Pre-Ex, samples taken before $2 \mathrm{~h}$ exercise bout; Post-Ex, samples taken immediately after $2 \mathrm{~h}$ exercise bout; $1 \mathrm{~h}$ Post-Ex, samples taken after $1 \mathrm{~h}$ of post-exercise recovery; PLA, placebo; COL, colostrum.

Post hoc analysis for time effects within the PLA group: mean values were significantly different from Pre-Ex within the PLA group ( $\left.{ }^{\star} P<0 \cdot 05\right)$. There were no differences in the $\mathrm{COL}$ group. Independent $t$ tests did not reveal any between-group differences at specific time points. 
shown beneficial effects on resting salivary $\operatorname{Ig} \mathrm{A}^{(11,12)}$ and upper respiratory tract infection incidence ${ }^{(13)}$ in athletes, none have investigated the effects on neutrophil function and innate salivary defences such as lysozyme. The present findings suggest that bovine COL may confer some benefits to innate host defence following prolonged exercise, but there were no effects on salivary IgA.

Shing et al. ${ }^{(14)}$ observed an enhancement effect of bovine COL on neutrophil surface receptor expression (CD89) at rest, which agrees with the trend for higher resting (Pre-Ex) neutrophil function in the present study. However, in the Shing et al. ${ }^{(14)}$ study, there were no differences in neutrophil CD11b, CD18 and CD35 expression. Although enhanced CD89 expression may represent enhanced microbicidal activity, no previous study has investigated a functional measure of neutrophil killing capacity (e.g. stimulated degranulation) in response to exercise. Furthermore, in the Shing et al. ${ }^{(14)}$ study, there were no differences in CD89 expression after exercise, possibly due to the relatively small changes that were actually observed acutely after exercise, even in the PLA condition. In the present study, when a significant decrease in neutrophil function was evident, neutrophil-stimulated degranulation was significantly higher at $1 \mathrm{~h}$ Post-Ex in the COL group. We believe that this is the first study to demonstrate that COL supplementation enhances neutrophil function in the recovery period after prolonged endurance exercise.

Many previous studies have examined the effect of prolonged exercise on neutrophils, demonstrating increased circulating neutrophil counts, depressed phagocytic activity and killing functions, such as oxidative burst and degranulation ${ }^{(20-32)}$. The findings of the present study are in line with these findings and confirm that a significant physiological stress was evident in both groups. There were significant exercise-induced increases is blood neutrophils, lymphocytes, neutrophil:lymphocyte ratio, immature granulocytes, atypical lymphocytes, plasma cortisol and blood lactate concentration, and a decrease in blood glucose, with no differences between the PLA and COL groups in the nature of these exerciseinduced changes. An increase in atypical lymphocytes may also be indicative of a physiological stress response ${ }^{(33)}$. This also confirms that participants were exposed to a matched physiological stressor and relative exercise stress. The magnitude of exercise-induced immunodepression is often proportional to the changes in circulating leucocytes, and the neutrophil to lymphocyte ratio, in particular, has been suggested as a marker of physiological stress to the immune system ${ }^{(34,35)}$. An increased release of immature cells from the bone marrow has also been suggested to contribute to decreased mean circulating neutrophil function post-exercise ${ }^{(20,22-24,36)}$. The increase in immature granulocytes observed in the present study provides support for this.

The fact that neutrophil degranulation was higher in the COL group at $1 \mathrm{~h}$ Post-Ex suggests that 'recovery' of this particular immune function may be speeded with COL supplementation. Inadequate recovery of the immune system (i.e. cumulative immunodepression ${ }^{(37)}$ ) can lead to significantly compromised immunity and increased risk in athletes. Li \& Cheng ${ }^{(27)}$ showed that a similar bout of exercise ( $2 \mathrm{~h}$ cycling at approximately $60 \% \mathrm{~V}_{\mathrm{O} 2 \max }$ ) caused a significant decrease in neutrophil functions (degranulation and oxidative burst), which persisted for at least $6 \mathrm{~h}$ post-exercise.
It is possible, therefore, that bovine COL supplementation can reduce this recovery period, but further study, with a more prolonged recovery period, is required in order to fully determine the extent to which COL enhances the recovery from exercise-induced immunodepression. As there was no difference between groups in any of the leucocytosis responses, it is likely that the effects of COL are related to direct effects on leucocytes, as observed in vitro by Sugisawa et al. ${ }^{(16,17)}$. It is worthy of note that Robson et al. ${ }^{(31)}$ observed enhanced neutrophil oxidative burst capacity, under resting conditions, in a group of athletes after $21 \mathrm{~d}$ of daily supplementation with a multivitamin and mineral supplement. The multi-nutrient supplement was administered to ensure that the recommended daily allowances were achieved and prevented any micronutrient deficiencies. It is possible, therefore, that the effects in the present study were related to micronutrients present in bovine COL. Bovine COL also contains antioxidants (such as vitamins $\mathrm{A}$ and $\mathrm{E})^{(16)}$, and it is a limitation of the present study that plasma antioxidant status was not assessed. However, findings from our previous studies ${ }^{(20,23)}$ suggest that this is an unlikely mechanism for the effects observed with bovine COL supplementation in the present study. For example, we have previously demonstrated that 2-4 weeks of dietary antioxidant supplementation ${ }^{(20,23)}$ does not influence neutrophil degranulation at rest or in response to exercise similar to that employed in the present study. The lack of difference, in the present study, between groups in the cortisol response (which may be influenced by antioxidant status $\left.{ }^{(20,22,23,28)}\right)$ also provides support for this argument. Furthermore, we have previously shown that supplementation of antioxidants for a 4-week period has little effect on overall plasma antioxidant capacity, presumably because antioxidant ingestion over such a time period results in down-regulation of natural endogenous antioxidant defences in response and/or adaptation to the higher intake of exogenous antioxidant compounds, so that total antioxidant capacity is maintained fairly constant ${ }^{(23)}$. These findings, taken together with the fact that there were no deficiencies in the normal diet of all subjects, and no differences between groups in the cortisol, glucose or leucocytosis responses, suggest that the effects are most likely due to other (bioactive) components present in COL. Strong evidence for this is provided by the work of Sugisawa et al. ${ }^{(17)}$, who showed that low molecular weight $(<10 \mathrm{kDa})$ components of COL directly prime and enhance neutrophil functions. Sugisawa et al. ${ }^{(17)}$ were not able to identify a specific compound, although suggested the proteose peptones as potential candidates. As an explanation for the present findings, this remains speculative and further research is required to determine whether this is the mechanism by which oral supplementation with bovine COL enhances innate immunity after physical stress in human subjects. It is also worthy of note that, in the present study, we only assessed one aspect of neutrophil function. In order to determine the mechanisms of action, future studies should also examine other aspects of neutrophil function, such as chemotactic, phagocytic and oxidative burst responses for example.

In the present study, there were no differences between groups for any of the salivary $\operatorname{IgA}$ parameters. Most notably, the supplementation did not cause an increase in resting IgA concentration or release, which does not agree with previous 
research $^{(11,12)}$. However, in the Crooks et al. ${ }^{(11)}$ study, supplementation comprised of $26 \mathrm{~g}$ of bovine COL per day for 12 weeks. In the Mero et al. ${ }^{(12)}$ study, supplementation comprised of $20 \mathrm{~g}$ of a bovine COL product per day for 2 weeks. However, this was divided into four equal doses spread throughout the day. Taken together, these findings suggest that a period of supplementation of longer than 4 weeks and/or dividing the dose throughout the day is necessary to cause significant changes in resting salivary $\operatorname{IgA}$. Indeed, Crooks et al. ${ }^{(11)}$ did not observe any differences between groups after 4 and 8 weeks of supplementation and a difference was only evident after 12 weeks.

Salivary lysozyme concentration and secretion rate were significantly reduced in the PLA group post-exercise, and had 'recovered' after $1 \mathrm{~h}$ of rest. We are not aware of any other study that has demonstrated an acute decrease of salivary lysozyme after prolonged exercise of this nature, and this may account for the observations of West et al. ${ }^{(38)}$ of chronically lowered salivary lysozyme in trained rowers. In the present study, the acute 'open window' of decreased salivary lysozyme, evident in the PLA group, was not evident in the COL group. It has been suggested that enhanced salivary IgA, when consuming bovine COL, may be responsible for protecting athletes from upper respiratory tract infection (self-reported symptoms) ${ }^{(13)}$. However, the present findings suggest that enhanced components of the innate immune system (neutrophil function and salivary lysozyme) may also contribute to host protection. This is in line with the suggestion of Shing et al. ${ }^{(14)}$, but the nature of the relationships between infection risk and aspects of mucosal immunity other than $\operatorname{IgA}$ (such as antimicrobial peptides) requires further study ${ }^{(18)}$.

In conclusion, 4 weeks of daily supplementation with bovine COL enhances the recovery of neutrophil function and prevents the decrease in salivary lysozyme release, but not $\mathrm{IgA}$, under conditions of exercise-induced immunodepression. This is the first study to demonstrate that COL supplementation enhances innate immune parameters in the recovery period after prolonged endurance exercise that causes significant immunodepression, and supplementation may confer some benefits to host defence.

\section{Acknowledgements}

The author contributions were as follows: G. D. was responsible for the conception of the study, and G. D. and B. C. D. produced the original study design, performed the laboratory investigations and biochemical analyses. G. D. undertook the statistical data analysis and interpretation. G. D. and B. C. D. wrote, read and approved the final manuscript. None of the authors had a conflict of interest. The authors would like to thank Ruth Hughes for management of the supplementation blinding procedures. Sources of support: The present study received partial financial support from Neovite UK, London, who also donated the bovine COL.

\section{References}

1. Gleeson M \& Pyne DB (2000) Special feature for the Olympics: effects of exercise on the immune system: exercise effects on mucosal immunity. Immunol Cell Biol 78, 536-544.
2. Hoffman-Goetz L \& Pedersen BK (1994) Exercise and the immune system: a model of the stress response? Immunol Today 15, 382-387.

3. Pedersen BK, Kappel M, Klokker M, et al. (1994) The immune system during exposure to extreme physiological conditions. Int J Sports Med 15, Suppl., S116-S121.

4. Pedersen BK, Bruunsgaard H, Klokker M, et al. (1997) Exercise-induced immunomodulation - possible roles of neuroendocrine and metabolic factors. Int J Sports Med $\mathbf{1 8}$, Suppl. 1, S2-S7.

5. Pedersen BK, Bruunsgaard H, Jensen M, et al. (1999) Exercise and the immune system - influence of nutrition and ageing. J Sci Med Sport 2, 234-252.

6. Pedersen BK \& Hoffman-Goetz L (2000) Exercise and the immune system: regulation, integration, and adaptation. Physiol Rev 80, 1055-1081.

7. Kelly GS (2003) Bovine colostrums: a review of clinical uses. Altern Med Rev 8, 378-394.

8. Pakkanen R \& Aalto J (1997) Growth factors and antimicrobial factors of bovine colostrum. Int Dairy J 7, 285-297.

9. He F, Tuomola E, Arvilommi H, et al. (2001) Modulation of human humoral immune response through orally administered bovine colostrum. FEMS Immunol Med Microbiol 31, 93-96.

10. Biswas P, Vecchi A, Mantegani P, et al. (2007) Immunomodulatory effects of bovine colostrum in human peripheral blood mononuclear cells. New Microbiol 30, 447-454.

11. Crooks CV, Wall CR, Cross ML, et al. (2006) The effect of bovine colostrum supplementation on salivary $\operatorname{IgA}$ in distance runners. Int J Sport Nutr Exerc Metab 16, 47-64.

12. Mero A, Kähkönen J, Nykänen T, et al. (2002) IGF-I, IgA, and IgG responses to bovine colostrum supplementation during training. J Appl Physiol 93, 732-739.

13. Brinkworth GD \& Buckley JD (2003) Concentrated bovine colostrum protein supplementation reduces the incidence of self-reported symptoms of upper respiratory tract infection in adult males. Eur J Nutr 42, 228-232.

14. Shing CM, Peake J, Suzuki K, et al. (2007) Effects of bovine colostrum supplementation on immune variables in highly trained cyclists. J Appl Physiol 102, 1113-1122.

15. Albers R, Antoine JM, Bourdet-Sicard R, et al. (2005) Markers to measure immunomodulation in human nutrition intervention studies. Br J Nutr 94, 452-481.

16. Sugisawa H, Itou T, Ichimura Y, et al. (2002) Bovine milk enhances the oxidative burst activity of polymorphonuclear leukocytes in low concentrations. J Vet Med Sci 64, 1113-1116.

17. Sugisawa H, Itou T, Saito M, et al. (2003) A low-molecularweight fraction of bovine colostrum and milk enhances the oxidative burst activity of polymorphonuclear leukocytes. Vet Res Commun 27, 453-461.

18. Davison G, Allgrove J \& Gleeson M (2009) Salivary antimicrobial peptides (LL-37 and alpha-defensins HNP1-3), antimicrobial and IgA responses to prolonged exercise. Eur $J$ Appl Physiol 106, 277-284.

19. Dill DB \& Costill DL (1974) Calculation of percentage changes in volumes of blood, plasma, and red cells in dehydration. J Appl Physiol 37, 247-248.

20. Davison G, Gleeson M \& Phillips S (2007) Antioxidant supplementation and immunoendocrine responses to prolonged exercise. Med Sci Sports Exerc 39, 645-652.

21. Bishop NC, Walsh NP \& Scanlon GA (2003) Effect of prolonged exercise and carbohydrate on total neutrophil elastase content. Med Sci Sports Exerc 35, 1326-1332.

22. Davison G \& Gleeson M (2005) Influence of acute vitamin C and/or carbohydrate ingestion on hormonal, cytokine, and immune responses to prolonged exercise. Int J Sport Nutr Exerc Metab 15, 465-479. 
23. Davison G \& Gleeson M (2006) The effect of 2 weeks vitamin C supplementation on immunoendocrine responses to $2.5 \mathrm{~h}$ cycling exercise in man. Eur J Appl Physiol 97, 454-461.

24. Davison G \& Gleeson M (2007) The effects of acute vitamin C supplementation on cortisol, interleukin-6, and neutrophil responses to prolonged cycling exercise. Eur J Sport Sci 7, $15-25$.

25. Li T-L \& Gleeson M (2004) The effect of single and repeated bouts of prolonged cycling on leukocyte redistribution, neutrophil degranulation, IL-6, and plasma stress hormone responses. Int J Sport Nutr Exerc Metab 14, 501-516.

26. Li T-L \& Gleeson M (2005) The effects of carbohydrate supplementation during the second of two prolonged cycling bouts on immunoendocrine responses. Eur J Appl Physiol 95, 391-399.

27. Li T-L \& Cheng PY (2007) Alterations of immunoendocrine responses during the recovery period after acute prolonged cycling. Eur J Appl Physiol 101, 539-546.

28. Peake JM (2002) Exercise-induced alterations in neutrophil degranulation and respiratory burst activity: possible mechanisms of action. Exerc Immunol Rev 8, 49-100.

29. Pyne DB, Baker MS, Smith JA, et al. (1996) Exercise and the neutrophil oxidative burst: biological and experimental variability. Eur J Appl Physiol 74, 564-571.

30. Robson PJ, Blannin AK, Walsh NP, et al. (1999) Effects of exercise intensity, duration and recovery on in vitro neutrophil function in male athletes. Int J Sports Med 20, 128-135.
31. Robson PJ, Bouic PJD \& Myburgh KH (2003) Antioxidant supplementation enhances neutrophil oxidative burst in trained runners following prolonged exercise. Int J Sport Nutr Exerc Metab 13, 369-381.

32. Scharhag J, Meyer T, Gabriel HH, et al. (2002) Mobilization and oxidative burst of neutrophils are influenced by carbohydrate supplementation during prolonged cycling in humans. Eur J Appl Physiol 87, 584-587.

33. Simon MW (2003) The atypical lymphocyte. Int Pediatr 18, $20-22$

34. Lancaster GI, Jentjens RL, Moseley L, et al. (2003) Effect of pre-exercise carbohydrate ingestion on plasma cytokine, stress hormone, and neutrophil degranulation responses to continuous, high-intensity exercise. Int J Sport Nutr Exerc Metab 13, 436-453.

35. Nieman DC, Nehlsen-Cannarella SL, Fagoaga OR, et al. (1999) Immune response to two hours of rowing in elite female rowers. Int J Sports Med 20, 476-481.

36. Berkow RL \& Dodson RW (1986) Purification and functional evaluation of mature neutrophils from human bone marrow. Blood 68, 853-860.

37. Lancaster GI, Halson SL, Khan Q, et al. (2003) Effect of acute exhaustive exercise and a 6-day period of intensified training on immune function in cyclists. $J$ Physiol 548P, O96, (abstr).

38. West NP, Pyne DB, Kyd JM, et al. (2008) The effect of exercise on innate mucosal immunity. Br J Sports Med (Epublication ahead of print version 22 May 2008) 